\title{
More uncertainty for the common man
}

\section{J.S. Bell}

THE aim of this book is to "face squarely the full impact of fundamental quantum theory on our conception of the world [and] not simply to review a notoriously difficult branch of modern physics, but to turn instead to broader issues. What is man? What is the nature of reality? Is the Universe we inhabit a random accident or the outcome of a delicate selection process?". And all this for a reader with "no previous knowledge of science or philosophy'. Professor Davies does not lack ambition.

In fact, I doubt that the book will mean much to a scientific ignoramus; it is more for a reader whose mind is not quite blank before words like 'electromagnetic', 'atom', 'galaxy' and so on. But, with just a minimum of basic scientific culture, it will be possible to get from the book some idea of what quantum theory is, of what an enormous break it is seen to be with what went before, and of how physicists have not yet really digested it.

The first chapters take the reader for a race, a bit breathless, through some of the main ideas of classical mechanics, relativity and quantum theory. We are told of how physicists have come to think of the world as a "game of chance in which we are not merely spectators but players" - in that we now have fundamental laws which are indeterministic in character and whose formulation (in the orthodox contemporary view, in so far as it is coherent) seems to require reference to the observer; of the uncertainty principle and the exclusion principle; of the Dirac sea and the antiparticle; of how space-time itself must be subject to quantum fluctuations and be 'foamy' on a small scale. Repeatedly we are told how very bizarre and incomprehensible it all is.

In later chapters the story unfolds of the remarkable ideas advanced by remarkable physicists in trying to comprehend it all Bohr's idea that we must renounce all attempts to visualize the atomic and subatomic world and must be content with a formalism that allows statistical predictions to be made about macroscopic events in macroscopic equipment; Wigner's concept that human consciousness plays an essential dynamical role in the world, in 'reducing the wave function'; Wheeler's idea that the very existence of the world, at any time, may somehow be caused by the existence of an observer at some time; the 'anthropic principle' of Dicke and Carter, that much in the observed world is fixed by
Other Worlds: Space, Superspace and the Quantum Universe. By Paul Davies. Pp.208. (Dent: 1980.) £7.50.

the fact that it is being observed, because with even minor changes the world could not have produced observing creatures like us.

Above all we learn of the 'manyuniverses' interpretation of quantum mechanics, of Everett and De Witt, according to which all universes that might exist do exist. If they all exist, then the existence among others of relatively few tailored for our comfort is no mystery, and of course it is only in one of these few that we could find ourselves. Professor Davies is clearly attracted to the many-universes interpretation - and derives from it the title of his book - but correctly makes clear that it is taken seriously only by a handful of physicists. A final chapter dwells on the mystery of time, and especially the absence from our equations of anything corresponding to our impression of the passing of time. One must admire the intellectual intrepidity, and subtlety, of the author in "finding no reason to suppose that the future does not already exist, even though it is not yet determined, and even though the observer will have a hand in structuring it ...".

All this is vividly done. Perhaps even a little too vividly. Sometimes the confidence, even the complacency, of the assertions can be irritating. "We must acknowledge ..." and "We have seen that ..." really mean only that some qualitative considerations might suggest the entertainment of one possibility among others. But often the unqualified statement is modified a little later, or even has been qualified earlier - a distinction perhaps of little moment to one with such an Olympian view of time. In the closing sentences of his book the author allows that our subjective impression might be more reliable as regards time's passing than our equations. And his assertion that most scientists would agree that "... past, present, and future are merely linguistic conventions ..." has been qualified already in the prologue, where it is said of such ideas that "most scientists live a sort of dual life, accepting them in the laboratory, but rejecting them without thought in daily life". Yes indeed.

On the whole the book gives a good impression of what is thought about these questions by physicists who consider them. But there is an exception, and it is a serious one. It concerns the views of Einstein in general and the Einstein-Podolsky-Rosen argument in particular. As regards Einstein's general reservations about quantum mechanics, Professor Davies propagates the view that they centred on indeterminism: "So unpalatable did this inherent chanciness seem to Albert Einstein that he refused to believe it throughout his life, dismissing the idea with the famous retort 'God does not play dice",". Perhaps the quotability of this famous retort is partly to blame for the fact that Einstein's more considered views are less well known. Compare the summing-up above with the following by Pauli. $\mathrm{He}$ is writing to Born in 1954:

Einstein does not regard the concept of 'determinism' to be as fundamental as it is frequently held to be (as he told me emphatically many times) ... he disputes that he uses as a criterion for the admissibility of a theory the question: - Is it rigorously deterministic? - . . he was not at all annoyed with you, but only said you were a person who will not listen',

The Einstein-Podolsky-Rosen (EPR) argument comes up in Chapter 6: "So far we have deliberately been rather cavalier about notions like the real world. . . . In this chapter we shall face up squarely to the fundamental questions raised by the quantum revolution... ". In particular, we face up squarely to the possibility that "the apparently capricious and random fluctuations of microsystems do not represent an intrinsic indeterminacy in Nature, but are simply manifestations of a hidden level of structure ... ". And then the EPR correlations are presented as the fundamental argument against such a possibility! While for EPR they were an entirely convincing argument for precisely such a possibility! The discussion exhibits the perplexity of one who refuses to contemplate the possibility that quantum mechanical description, of a photon in particular, is incomplete. It was the position of EPR that such perplexity could be relieved just by admitting the hypothesis in question. That Professor Davies misses the point not only of the original work, but of the renewed discussion and the associated experimental programme, is clear from the following: "The truly mind-boggling implications . . . are apparent if we use two parallely orientated polarizers ...'”. On the contrary, the mind is boggled only by 
polarizers which are not parallel. The results with parallel polarizers are easily explicable along Einsteinian lines. The results with off-parallel polarizers are not. That is the new point, unknown to EPR, which inspired the experimental programme of recent years to which the author seems to refer.

While I claim that Professor Davies is wrong on these points, it would certainly be unjust to pillory him on account of them. He makes these mistakes, if they are indeed mistakes, in the company of many other distinguished physicists. He could claim to be reporting a consensus! I enjoyed the book, despite all the reservations, and expect it to be enjoyed by many. The author provides a lively account, accessible with very little technical background, of what is being said about some momentous and inevitable questions. And if he sometimes leads into error, he does not lead into a minority.

J. S. Bell is a physicist working at CERN in Geneva.

\section{Data book on zinc}

\section{Roy M. Harrison}

Zinc in the Environment. Edited by J. O. Nriagu. Part 1 Ecological Cycling, pp.453; Part 2 Health Effects, pp.480. (WileyInterscience: 1980.) Each volume $£ 27.50$, $\$ 59.85$.

MOST non-specialists will probably associate the subject of metals in the environment with possible adverse human health effects; indeed in the cases of metals such as lead, cadmium and mercury the main stimulus for research has arisen from concerns over toxic symptoms arising from excessive environmental exposure. Zinc provides a very different case, however. Adverse health effects found in the general population result more often from zinc deficiency, rather than from toxicity due to elevated exposure. Indeed, despite the ubiquitousness of zinc in our environment, numerous well-documented cases of serious zinc deficiency syndrome have arisen amongst groups of people in many different parts of the world. Instances of zinc intoxication in humans are comparatively rare, arising only from extreme exposure conditions not normally experienced by the general population, and in some instances probably due to the cadmium impurity invariably present in technical-grade zinc.

The essentiality of zinc in mammalian metabolism coupled with its considerable toxicity, especially to aquatic biota, make the study of zinc in the environment an important matter which has been rather neglected in comparison with the intensive research efforts expended upon other environmental metals. This two-volume compilation by Nriagu may help to redress the balance; it is an extremely valuable collection of both factual information and numerical data on environmental zinc, and provides a useful source book

Part I covers the production and uses of zinc, the environmental concentrations and pathways of the metal and its cycling within, and effects upon, near-shore marine, freshwater and forest ecosystems. Part II deals primarily with human health effects of zinc, but also includes the effects of zinc upon terrestrial and aquatic flora and fauna.
As in other recent books edited by Nriagu, the emphasis is upon detailed, but frequently uncritical reviews of the literature. Contributions are drawn from a large number of specialist authors and are fairly well up to date, with references up to 1978 included. The editor has imposed a fairly high degree of uniformity of style, and the book gives wide coverage of the topic with few omissions or overlaps. In these regards the book is of far greater value to the reader than the now-common review volumes cobbled together from the proceedings of a symposium and purporting to provide complete coverage of a topic.

Zinc in the Environment cannot be recommended as light reading for the individual requiring a brief overview of the subject. It will, however, be regarded as essential by many research workers in the field and will, in my view, represent an invaluable source of information for a good many years to come.

Roy M. Harrison is a Lecturer in the Department of Environmental Sciences at the University of Lancaster.

\section{En route to a classic of ornithology}

\section{Raymond O'Connor \\ Handbook of the Birds of Europe, the Middle East and North Africa: The Birds of the Western Palearctic. Vol.II. Edited by Stanley Cramp. Pp.695. (Oxford University Press: 1980.) $£ 30, \$ 85$.}

THIS is the second volume of The Birds of the Western Palearctic, the ambitious multi-volume work intended as the successor to Witherby's Handbook of British Birds, though with a wider geographical brief than the British Isles of the 1939 work.

The success of Vol.I testifies to the overwhelming acceptance of its general approach and format, and Vol.II follows the same pattern. The volume treats those species belonging to the Accipitriformes, Falconiformes, Galliformes and Gruiformes. Each species account begins with a general consideration of field characters followed by detailed discussion

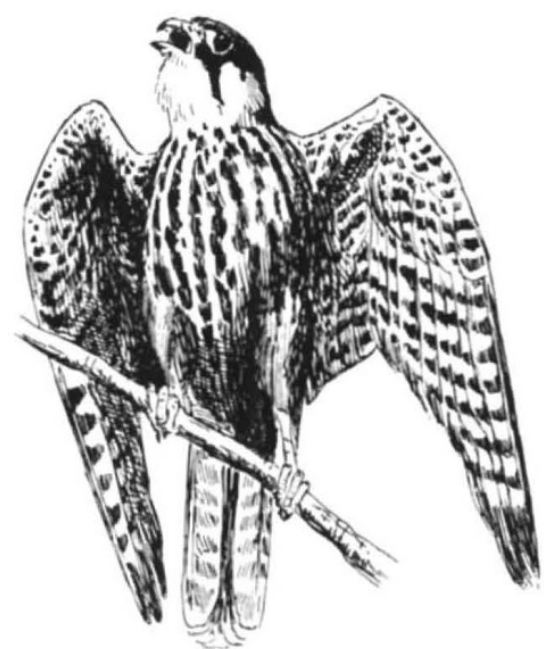

of the plumage characteristics of the recognizable age-classes. Sections on habitat, distribution, population, movements, food, behaviour and breeding follow, and each account is concluded with a listing of plumage and morphology data. The approach is entirely specific except for brief introductions to each family. The editors have rightly persisted with the format of Vol.I with only minor modifi- cations, though I wish they would quote author initials when citing authors of the same surname. Another minor irritant is the failure to reproduce the explanatory material given in the first volume. As a reference work the Handbook should not require one to consult two hefty tomes to locate one's target information within Vol.II.

One general feature of this work is that its treatment of a topic is determined very much by the nature of the material available. For the kestrel, Falco tinnunculus, for example, nest site frequencies are tabulated for German and British studies, regional differences are described between northern and southern Britain, seasonal variation in clutch size is cited on the basis of a Swiss study, egg loss rates are quantified for Dutch and English populations, and breeding success to food supply correlations are mentioned for a Norwegian study. With the Western Palearctic as their geographical remit, the authors are entitled to their use of studies from different areas but I find two shortcomings in this approach. First, is the reader to assume that effects cited in a regional study are common to the whole 